NASATM. $-76-208064$

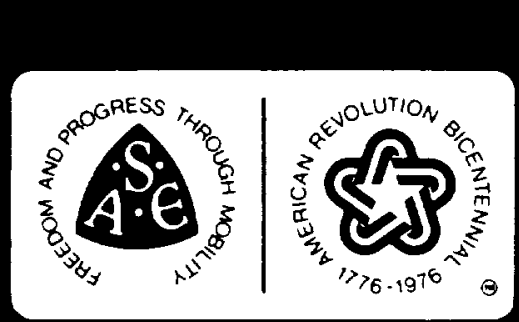

SOCIETY OF AUTOMOTIVE ENGINEERS, INC. 400 Commonwealth Drive, Warrendale, Pa. 15096

\title{
Reduction of Stall-Spin Entry Tendencies Through Wing Aerodynamic Design
}

\author{
R. A. Kroeger \\ The University of Michigan \\ T. W. Feistel \\ NASA Ames Research Center
}

\section{SOCIETY OF AUTOMOTIVE ENGINEERS}

Business Aircraft Meeting Wichita, Kansas April 6-9, 1976
760481 


\section{Reduction of Stall-Spin Entry Tendencies Through Wing Aerodynamic Design}

POST STALL DYNAMICS OF AIRCRAFT can produce uncontrollable motions which are often accompanied by high yawing and rolling rates. If the longitudinal pitching moment is nose up, these initial motions are called incipient spins. If the pitching moment is nose down, they are called incipient spirals. In any event, should such "departures" occur at relatively low altitudes, say traffic pattern height, it becomes difficult for the pilot to make adequate control adjustments. So-called "stall/spin" accidents are one of the greatest causes of fatalities in light aircraft. Engine failures on take-off, for example, are of ten followed by aircraft gyrations which inadvertently precipitate severe wing lift loss. The propagation of the spanwise load changes on the stalling wing(s) is significant in the ensuing dynamics.

This paper describes research which is to provide a logical basis for, and to demonstrate a method of developing relieving wing span-loadings which lead to a reduction in the post-stall departure forces. (U1timately, of course, the research must include an analysis
R. A. Kroeger

The University of Michigan

T. W. Feistel

NASA Ames Research Center of the complete airplane dynamics problem to be valid.)

\section{BACKGROUND}

The parameters which directly influence the post stall handling qualities and departure resistance of afrcraft are listed below, from Ref. (1)*.

1. Lift Curve Top, $\mathrm{C}_{\mathrm{L}}$ versus $\alpha$

2. Rolling Moment, $\mathrm{C}_{1}$

3. Roll Damping, $\mathrm{C}_{1_{\mathrm{p}}}$

4. Roll Control Power, $\mathrm{C}_{1} \delta_{\mathrm{A}}$

5. Aileron Adverse Yaw, $\mathrm{CN}_{\delta_{\mathrm{A}}}$

6. Directional Stability, $\mathrm{C}_{\mathrm{N}}^{\mathrm{A}}$

7. Yaw Damping, $\mathrm{C}_{\mathrm{r}}$

8. Dihedral Effect, $\mathrm{C}_{1 \beta}$

9. Side Force, $C_{Y}$, and Yawing Moment, $\mathrm{C}_{\mathrm{N}}$

10. Yawing Moment Due to Rolling, $\mathrm{C}_{\mathrm{NP}}$

11. Pitching Moment, $\mathrm{C}_{\mathrm{m}}$

In general, the post-stall wing performance affects each of these parameters; some

*Numbers in parentheses designate References at end of paper.

\section{ABSTRACT}

Results of recent wind tunnel tests and analyses are described which are almed at helping to prevent spin entry after a stall by modifications to the aircraft wing. Simple add-on devices were ut1lized which modify the shape of the lift curve after stall, making it essentially flat to $30-40^{\circ}$ angle of attack. It is hypothesized that this will greatly reduce the danger of spin entry. Further research will be almed at laying a firmer theoretical and analytical basis for the approach and at incorporating the complete full scale alrplane and 1ts dynamics into the problem. 
to a greater extent than the others. However, if the post-stall change in wing span loading could be designed to be gradual and nearly symmetrical about the alrcraft roll and yaw axes, very gentle adverse accelerations would occur. Also, if adequate stall warning and good post-stall control could be sustained the research objectives would be met. It may thus be argued that the first step in solving the departure problem is to cause the post-stall spanwise wing unloading to be symmetrical and gradual, even under the adverse condition of a severe, tip-to-tip angle-of-attack variation (this has been the primary object of the research conducted thus far). For example, if one wing were stalled at $\alpha=17^{\circ}$ (average), and the other at $\alpha=25^{\circ}$, no sudden roll accelerations should occur. This leads to the requirement for the so-called "flat top" lift curve. Now, if the drag difference between the two stalled wings is small and effective roll control without adverse yaw is available, many of the departureinducing derivatives are signiflcantly reduced. Though the shape of the three-dimensional wing lift curve proposed does not positively preclude fully developed spins (flat spins in particular) its development will certainly have a preventive influence on the initial post-stall acceleration rates and the pilot's lateral, directional, and longitudinal control authority. Several practical constraints are always imposed on the solutions and thus the direction of research conducted for generalized light aircraft. These include limitations of weight, cost, simplicity, durability, reliability, maintenance, manufacturing, performance and customer acceptance. Though flight safety is the ultimate goal of this research, only those solutions which satisfactorily meet the constraints are reported in this paper.

Finally, the material presented has not been verified by full scale testing as of this writing. Its significance, however, was felt to be sufficient to warrant publication at this time.

\section{TECHNICAL DISCUSSION}

A combined theoretical and experimental aerodynamic research effort has been conducted. The theoretical analysis was based on the work reported in Refs. (2) through (4). The experimental work was performed in The University of Michigan and NASA Ames low-speed wind tunnels. It has been partially documented in Refs. (5) and (6).

THEORETICAL APPROACH - Nonlinear lifting line theory was selected as the basis of the theoretical approach used in the research reported here. The primary constraint of the theory was that the post-stall nonlinear aerodynamic characteristics of the elements (chordwise truncated segments of the wing span) were known. More sophisticated nonlinear analyses have been reported, for example, Ref. (7). However, the extent of the development of Ref. (4) made the more simple approach most attractive.

In essence, the basis of the lifting line theory is as follows: If the wing may be represented by a set of bound vortex elements, having strengths which collectively provide the proper spanwise loading, then the local lift coefficient, $c_{\ell}\left(y, \alpha_{e}\right)$, chord, $c(y)$, and span $b$, produce a spanwise downwash along the lifting line. This downwash is given by;

$$
w(y)=\frac{v_{\infty}}{8 \pi} \int_{-b / 2}^{h / 2} \frac{d}{d y}\left[c_{\ell}\left(y^{\prime}, \alpha_{e}\right) c\left(y^{\prime} / b\right)\right] \frac{d y^{\prime}}{y-y^{\prime}}
$$

where $\alpha_{e}$ is the local effective angle-of-attack, $V_{\infty}$ is the free-stream velocity and $y$ and $y^{\prime}$ are the spanwise coordinates. The local effective angle-of-attack becomes;

$$
\begin{aligned}
\alpha_{\mathrm{e}}(\mathrm{y}) & =\alpha(\mathrm{y})-\tan ^{-1} \frac{\mathrm{w}(\mathrm{y})}{\mathrm{V}_{\infty}} \\
& \simeq \alpha(\mathrm{y})-\frac{\mathrm{w}(\mathrm{y})}{\mathrm{V}_{\infty}}
\end{aligned}
$$

and the local lift coefficient becomes

$$
c_{\ell}=f\left(\alpha_{e}\right)
$$

Similarly the remaining nonlinear load coefficlents may be calculated once the effective local angle of attack is known.

Figure 1 shows the types of section stall used in the analysis. Practically, the trailingedge stall is accompanied by gradual turbulent separation from the rear of the airfoil, leadingedge stall is initiated by a short bubble from the leading edge and thin airfoil stall with a long bubble. The limitations of the theory were clearly demonstrated when the post-stall lift characteristics were varied. A doctoral dissertation issued by The University of Michigan (8) provides the proof of the existence of a mathematical singularity which arises as a consequence of the post-stall negative lift-

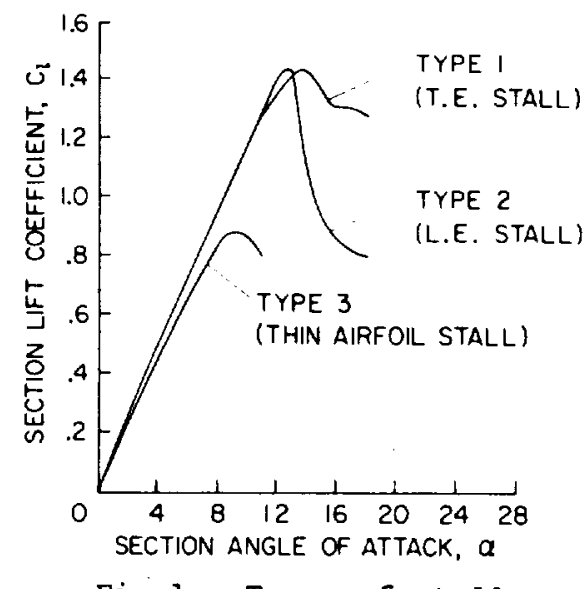

Fig.l - Types of stall 
curve slope. Qualitative post-stall results were obtained for the lifting line theory using a time dependent solution. In addition, the existence of 1 ift hysteresis was indicated. Such effects are well known in helicopter dynamics (9).

The results of the theoretical study may be summarized as follows:

1. The method of Refs. (2)-(4) is inadequate for general post-stall calculations.

2. Qualitative, 3-D results have been obtained using a "transient solution" approach.

3. Hysteresis in $\mathrm{C}_{\mathrm{L}}$ vs $\alpha$ does exist mathematically.

4. A nicely rounded (pure T.E. stall) section 1 ift curve does not preclude rapid poststall lift loss.

5. A more clear understanding of wing stall propagation is becoming avallable.

6. Stable asymmetrical lift solutions exist beyond stall.

Additional theoretical studies were made using the linear vortex-lattice method to determine the influence of the location of the stalled portion of the wing. Both span-wise and chordwise separation were simulated by allowing lift loss at the local "stalled" region. It was seen that local leading edge type separation produced a much stronger induced angle-of-attack distribution than trailing edge separation (6). This study led to the logic which ultimately guided the experimental program. Figure 2 shows how the trailing edge

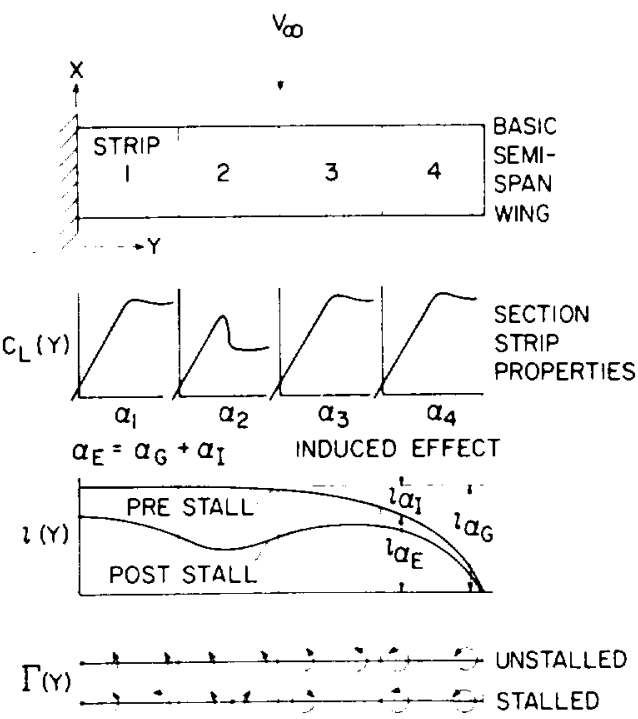

Fig. 2 - The stalling "strip" model

and leading edge separation were mixed to localize the wing stall propagation. In essence, the premature leading edge stall over a limited spanwise region unloaded the remainder of the wing by decreasing the adjacent geometric angle of attack. This was provided by the local induced angles-of-attack. Further, the stability studies of the non-linear lifting line theory implied the need for a local poststall positive lift curve slope (following the rapid lift loss initiated at stall) to limit the induced total wing lift loss. Figure 3

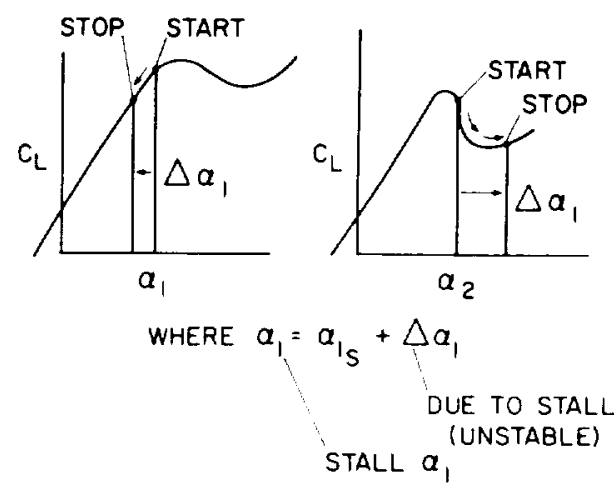

Fig. 3 - The strip theory stalling thesis (strips 1 and 2 from Fig. 2)

demonstrates this argument. As $\alpha_{2}$ becomes unstable, it continues to diverge in effective angle-of-attack until it reaches a positive lift curve slope. Simultaneously, $\alpha_{1}$ is diminished or maintained until the entire wing reaches a stable point. Thus, if the stalled region can be localized (and stabilized), higher geometric angles-of-attack of the wing can be achieved with a reduced wing lift loss rate, resulting in approximately constant $C_{L}$ to a high $\alpha$.

Lift hysteresis is also a consequence of the spanwise induced angle-of-attack instability. Figure 4 demonstrates how this can occur. After stall, the strip effective angle-of-attack continues to increase until a stable point is reached, well up on the post-stall positive lift slope. Then, as the geometric angle-of-attack is reduced, stability persists until the instability re-appears, though at a lower angle-ofattack than at the previous stable point. Again, this is due to the induced flow interactions between all spanwise elements of the wing. When the reattachment occurs, the geometric angle-of-attack can be below that of the initial stall. The inset "wing $C_{L}$ " curve in Fig. 4 shows the effect on the entire wing.

EXPERIMENTAL STUDIES - The experimental program was closely aligned with the theoretical studies. The main objective was to find a means for containing the separating region so that the flat-top lift curve could be produced. The approach taken was as follows:

1. Tuft studies for post-stall comparison of the wind tunnel model with a full scale flight test wing,

2. Flow visualization of upper surface flow to allow a qualitative understanding of the separation mechanics,

3. Investigation of devices to localize the separated flow,

4. Study wing hysteresis, 


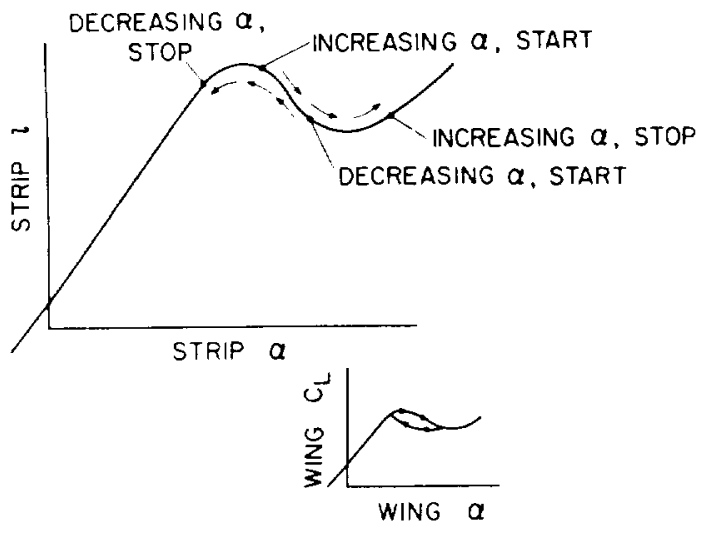

Fig. 4 - Stalling hysteresis illustration

5. Investigate the effect of mixing leading edge and trailing edge separation on a wing,

6. Develop a flat top lift curve, and

7. Improve performance.

The requirements of the research program as of this writing limited the studies to simple rectangular wings. In addition, retrofit devices were first studied because of their timeliness as defined by minimal manufacturing changes.

Two slightly twisted $\left(1.5^{\circ}\right)$, aspect ratio seven wings with an NACA $652-415$ airfoil were obtained from NASA Langley. One was modified for the University of Michigan's $5^{\prime} \times 7^{\prime}$ wind tunnel installation and the other for the NASA Ames $7^{\prime} \times 10^{\prime}$ wind tunnel. The wings were tufted to study the nature of the flow separation. At $\mathrm{C}_{\mathrm{L}_{\max }}$, the tufts exhibited moderate spanwise flow at the trailing edge (Fig. 5). As the angle-of-attack was increased slightly, leading edge (large bubble) separation occurred. The induced effects caused an upper surface stagnation vortex set to be shed. The strength of the cel1, illustrated in Fig. 6, implies the level of lift loss. Also, the weakness of the inboard induced counter-rotating cell set implies the absence of upper surface lift in that area. It was interesting to note the dynamics of the upper surface flow during the incipient stall. An instability was present which changed the tuft patterns alternately (and implicitly, the lift) between the pattern shown and one with the vortex cell moved further outboard. This instability caused significant model motions as well as changes in tunnel flow conditions. It was noted in the lift readout that more of a bi-stability as opposed to an instability was present. When the model angle-of-attack was increased the violent nature of this instability disappeared. Finally as the complete upper surface was stalled, though turbulence persisted, the wide ranging lift fluctuations disappeared.

Next, a series of photographs was taken to demonstrate the flow preference with increasing versus decreasing angles-of-attack. Figure 7 depicts the results. Note the inset sketch $\alpha=17^{\circ}$

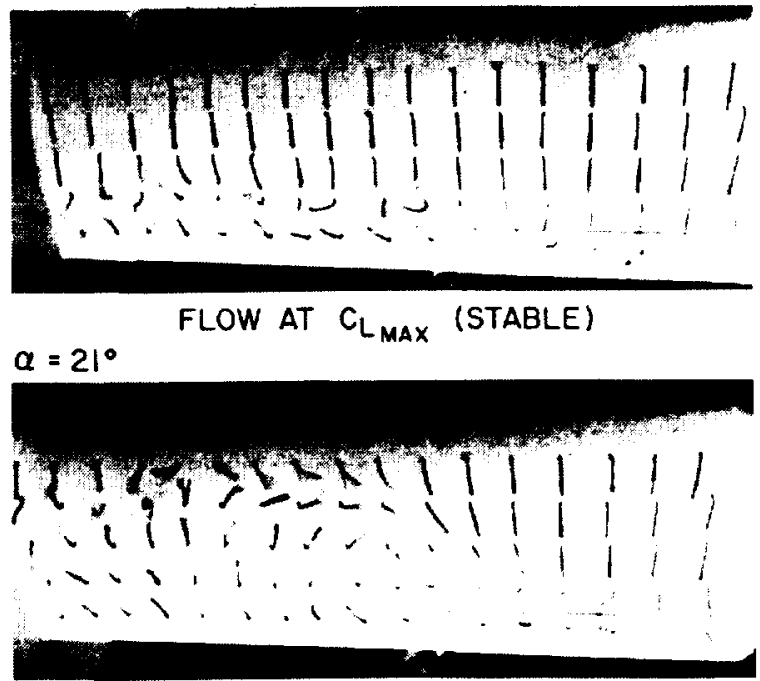

INCIPIENT STALL (UNSTABLE)

$\alpha=25^{\circ}$

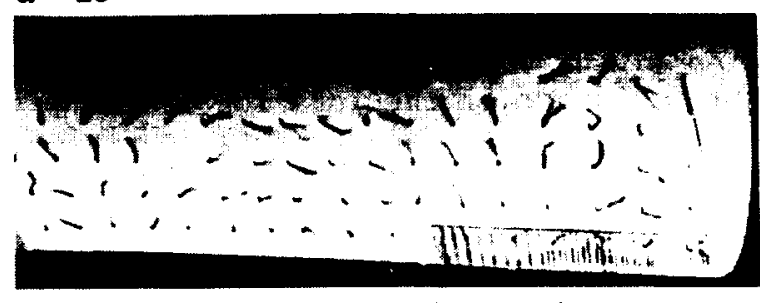

POST STALL (STABLE)

Fig. 5 - Stall progression, basic wing
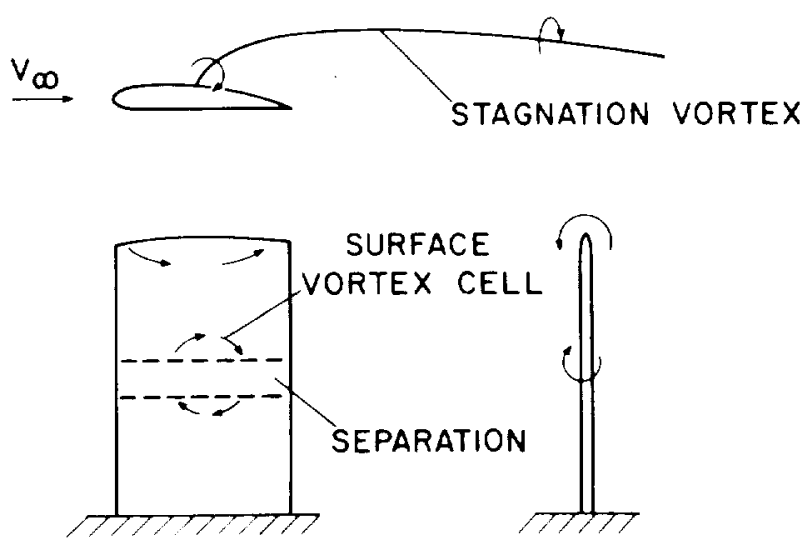

Fig. 6 - Example of a separation vortex

showing model attitude and wind direction. It became clear that with increasing angles-ofattack, at a moderate rate of approximately $2 / 10$ degrees per second, the lift chose the higher value. Read upper photos from left to right. As the angles-of-attack were reduced from 27 to 14 degrees, from right to left in the photos, a hysteresis was demonstrated. Between 18 and 22 degrees (stall being at approximately $17^{\circ}$, uncorrected) unique flow patterns developed.

A series of wind tunnel force measurement studies was next made to evaluate various types 


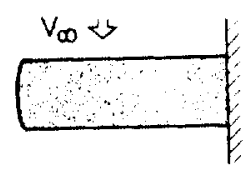

NOTE UNIQUE FLOW PATTERN WITH DECREASING $\alpha^{\prime} s 18,20 \& 22 \mathrm{deg}$

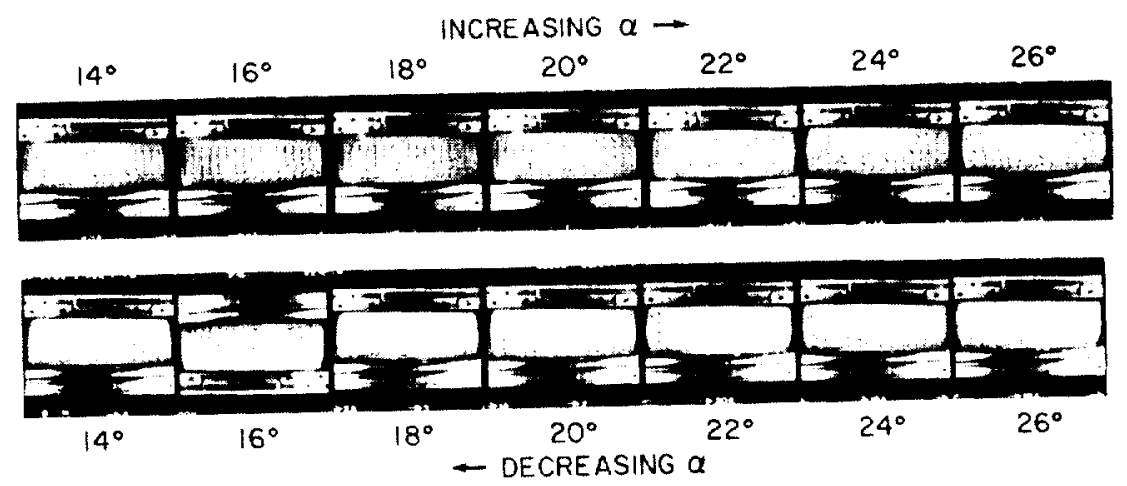

Fig. 7 - Demonstration of flow hysteresis

of separation stabilization devices. These included:

1. Various forms of vortex generators,

2. Fences, both streamwise and canted,

3. Flexible (floating) trailing edge devices, and

4. Various forms of leading edge vortex generators and stall strips.

The results were that all of these devices, when applied to a wing prone to leading edge separation, produced results which were far too subtle to provide improvements at high angles-of-attack.

Next, devices which would have a much stronger influence on the character of the flow separation were investigated. These included:

1. Leading edge slats, and

2. Drooped leading edges.

It is felt that a more superior method than either of the above is now available through Ref. (10). This approach uses localized thickening of the upper surface toward the leading edge. However, as will be shown, the two devices listed above were sufficient to demonstrate the flat-top lift curve desired. Later studies will include the approach of Ref. (10) as it provides a generalized flexibility, can produce better performance and blends well with the manufacturing requirements.

Figure 8 shows the nomenclature and describes the stripwise quartering of the leading edge devices used in the experimentation. Various combinations of leading edge device locations were attempted as a means of obtaining localized trailing edge separation. Tuft studies were used in the beginning and force measurements later. Also during the NASA Ames wind tunnel tests, one of the leading edge segments was split into two $121 / 2 \%$ span lengths (A \& B) for further refinement.

First, slats were used. The results showed significant promise as the stall could be tal-

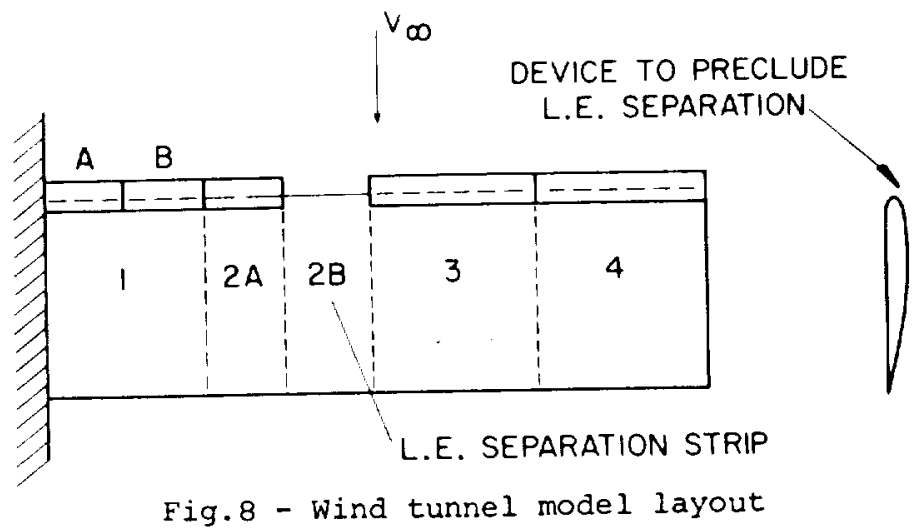

lored to cause a loss of only $5 \%$ of the lift and quickly resume a positive $\mathrm{dCL} / \mathrm{d} \alpha$ slope. This could be sustained to angles-of-attack in excess of $40^{\circ}$. The best location for the unslatted portion was position 2 as shown in Fig. 8. More details of the study are given in Ref. (6).

Next, drooped leading edge segments patterned after the GAW-1 airfoil (Fig. 9) were used. Since the upper surfaces of both airfoils are so similar, this approach promised good cruise performance and trailing edge separation in stall. Wind tunnel tests were made at NASA Ames to determine the best spanwise location and width of the undrooped section. The results showed location 2B(Fig. 8) to be the best position to remove the $121 / 2 \%$ drooped segment. The tuft photos of F1g. 10 show the significance of bounding the leading edge separation with trailing edge separation. Figure 11 further shows the stability of the flow pattern up to $40^{\circ}$ angle-of-attack. Comparison of the lift and drag data between the plain wing and configuration 2B is shown in Fig. 12. The hysteresis of the plain wing was obtained by averaging the data of several runs. Note the significant reduction in the pro-spin qualities of both $11 \mathrm{ft}$ 


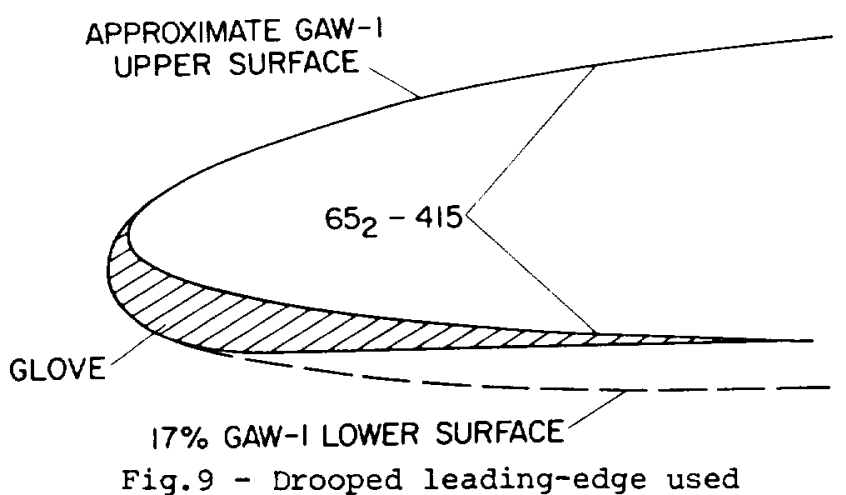

consequently, no lift carryover across the fuselage (with an attached region at midspan on each wing and generally, a separated region at the tip). These phenomena can be observed by careful study of the post-stall tuft photos for the 652-415 wing with and without the "2B configuration" L.E. devices.

The final wind tunnel tests of this series was for the evaluation of the stall characteristics of the $17 \%$, GAW-1 wing, rectangular planform, aspect ratio seven. The model was obtained from NASA, Langley and was identical in construction to the previously tested wing. The Reynolds

WING WITH 2-B MODIFICATION

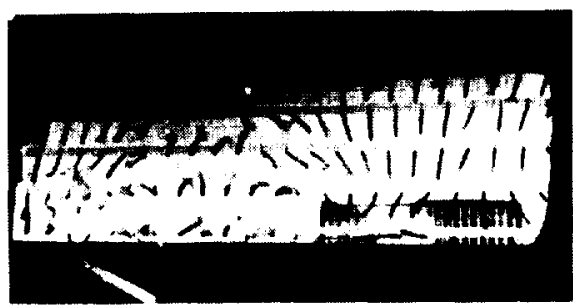

CLEAN $65_{2}-415$ WING

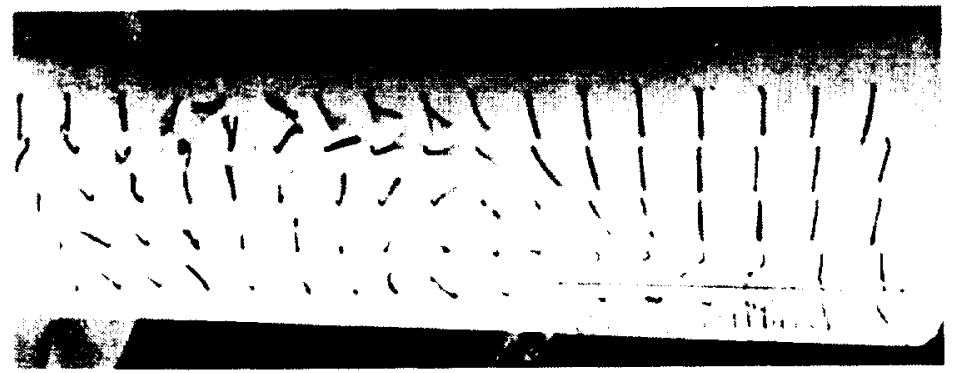

Fig. 10 - Comparison of wing flows at $\alpha=20^{\circ}$

and drag using the modified wing. Also, the flap top lift curve has been demonstrated beyond $30^{\circ}$ angle of attack. Equally as significant is the maintenance of lift effectiveness with alleron deflection. Figure 13 shows no severe $1 \mathrm{ift}$ loss and thus implies aileron effectiveness beyond $40^{\circ}$ angle-of-attack. The values of $15^{\circ}$ down and $30^{\circ}$ up aileron chosen are typical of fleet aircraft.

A simplistic explanation of the reason for the success of the "2B configuration" in producing a flattop lift curve is as follows: Moving the "L.E. stall strip" location outboard from the root allows a lift carryover to survive across the fuselage and produces three lifting cells across the span at high $\alpha^{\prime} s$ as opposed to the two normally achieved with the "stall first at the root" philosophy, i.e. the wing, at post stall $\alpha^{\prime} s$, is transformed into three low aspect ratio surfaces: one across the fuselage and on the wing roots, and one on each tip section (with a separated area in between). In contrast, the "conventional" stall pattern results in separation in the wing roots, and number was $2 \times 10^{6}$ in the Ames $7^{\prime} \times 10^{\prime}$ tunnel as before. Though the value of $\mathrm{C}_{\mathrm{L}_{\max }}$ for the wing was somewhat higher than the $652-415$, the post-stall lift curve was continuously diminishing and without the upturn at $30^{\circ}$ angle-of-attack The hysteresis levels appeared to be much lower as was the average rate of lift loss with angleof-attack. Several types of stall strips were used to induce leading edge separation in the same position, 2B. The results are shown in Fig. 14 for comparison with the 652-415 leading edge. An improvement in the flatness of the GAW-1 lift curve was attained with the stall strips though it was accompanied with a severe

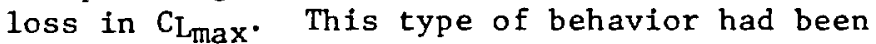
expected as a result of the analytical studies.

\section{CONCLUSIONS AND RECOMMENDATIONS}

The conclusions, based on the work to date may be summarized as follows:

1. The non-linear lifting line theory as classically presented is not generally useful for post-stall studies. 


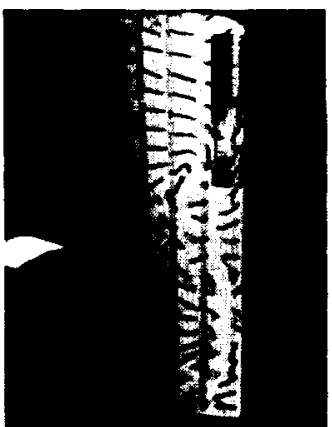

$\alpha=18^{\circ}$

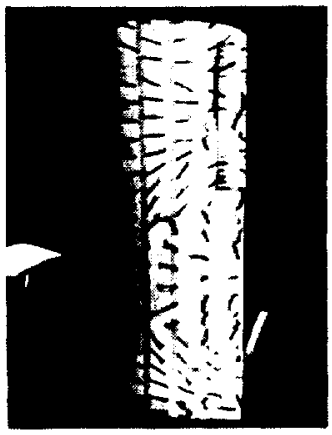

$\alpha=30^{\circ}$

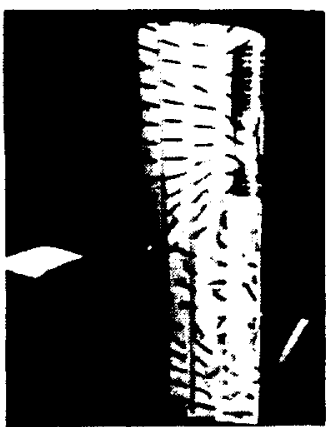

$\alpha=20^{\circ}$

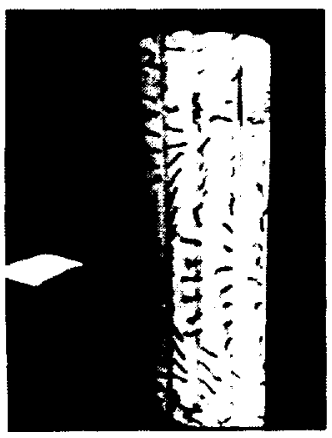

$\alpha=40^{\circ}$
Fig.11 - "Configuration 2B" showing separation localization at high angles-of-attack

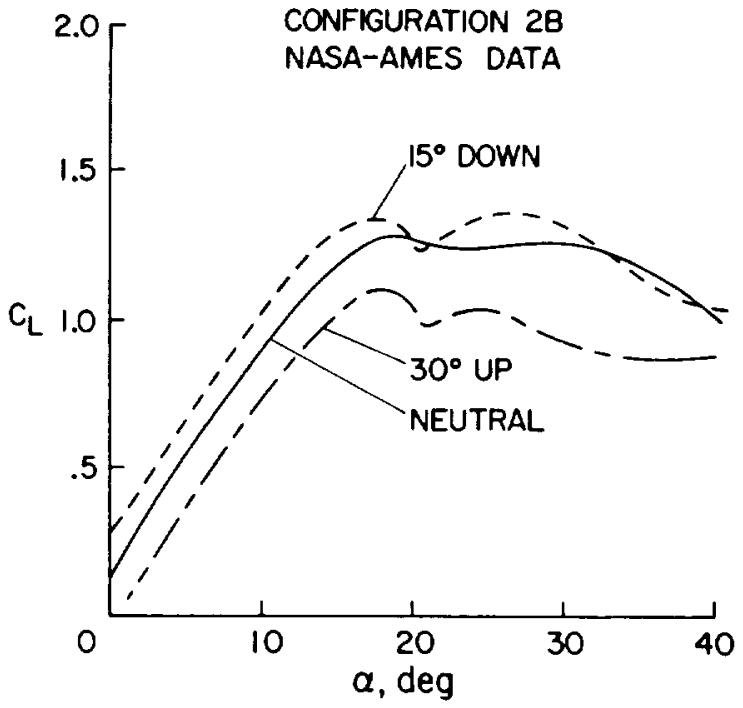

Fig.13 - Effect of aileron on "configuration 2B" lift curve

the untreated wing.

5. The pro-spin hysteresis effect has been diminished by proper mixing of leading and trailing edge stall.

6. The flat-top $11 \mathrm{ft}$ curve is accompanied by good lift and drag characteristics with post-

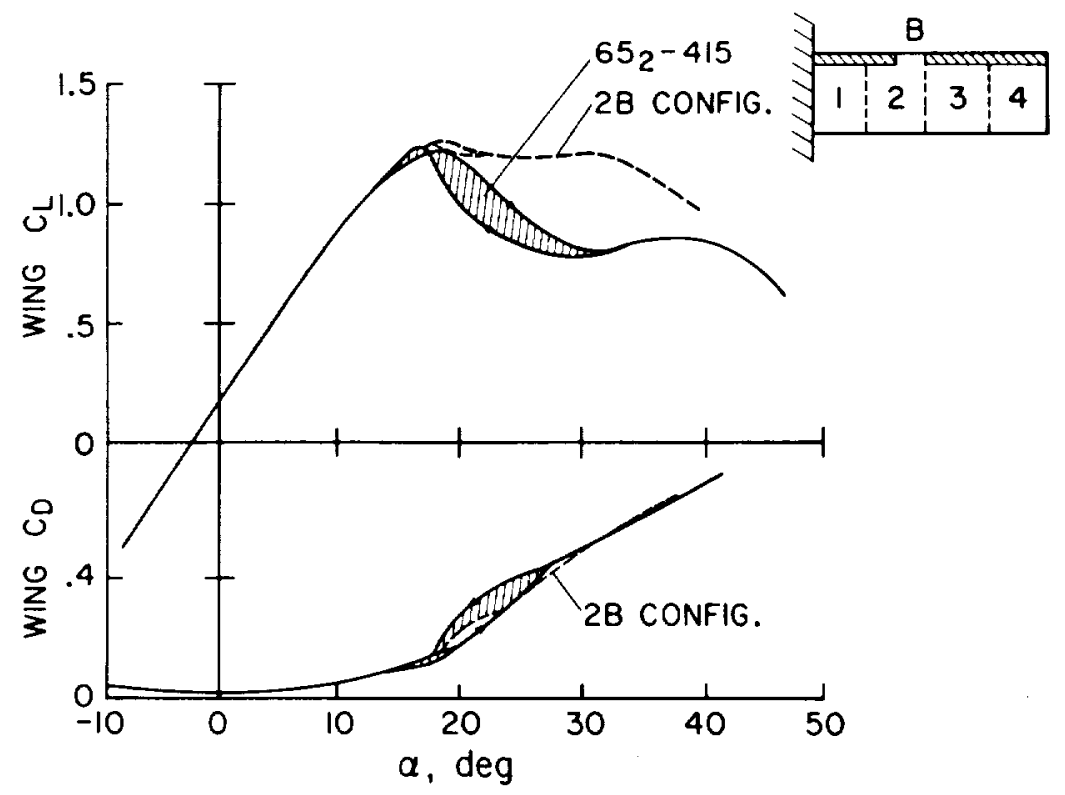

Fig.12 - Demonstration of "flat top" lift curve

2. Lifting line solutions have been extended beyond stall using a transient solution. 3. Hysteresis exists in lift and drag with angle-of-attack under the conditions observed.

4. An experimental method, based on the logic of an analytical model, has been developed for obtaining a "flat-top" lift curve with no loss in $C_{L \max }$ or measurable $C_{D}$ increase over stall aileron deflection.

7. A nicely rounded two-dimensional lift curve, accomplished with trailing edge separation, does not preclude rapid three-dimensional post-stall lift loss if the full span section is constant (6).

8. Mathematically, asymmetrical full span stable lift distributions exist beyond stall for simulated steady unaccelerated flight (6). 


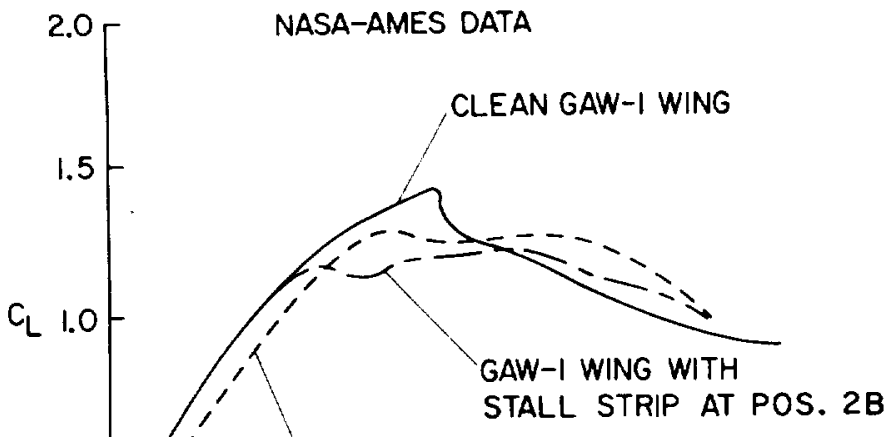

CONFIG. $2 B$

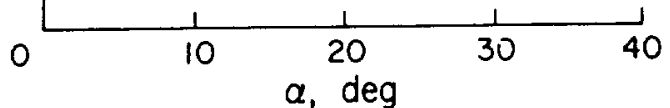

Fig.14 - Comparison with "GAW-1 wing" lift curves

It is anticipated that the research will be continued with the following objectives;

1. Develop a digital computer solution to predict the general post-stall wing load distribution for research and design purposes.

2. Extend the theoretical and experimental investigations to include wing geometric characteristics such as taper, twist, flaps, and sweep.

3. Apply the new concepts and theoretical modeling capability to the evaluation of incipient spin handling qualities.

4. Demonstrate the spin resistant qualities of the flat top lift curve by full scale tests.

The recommendations listed above are the goals of ongoing research being jointly approached by NASA Ames and The University of Michigan under NASA sponsorship.

\section{REFERENCES}

1. S.B. Anderson, "Design Considerations for Stall/Spin Avoidance," NASA, Ames working paper.

2. J.C. Sivells and R.H. Neely, "Method for Calculating Wing Characteristics by Lifting Line Theory Using Nonlinear Section Lift Data," NACA Report 865.

3. J.S. Sive11s and G.C. Westrick, "Method for Calculating Lift Distribution for Unswept Wings with Flaps or Allerons by use of Nonlinear Section Lift Data," NACA TR 1090, November 1950.

4. M.A. McVeigh and E. Kisielowski, "Design Sumnary of Stall Characteristics of Straight Wing Aircraft," NASA CR 1646.

5. H. Buning, "Graduate Research Program in Aeronautics on the Aerodynamics of Stall/ Incipient Spin," First Semi-Annual Status Report, NASA Grant NSG-2018, The University of Michigan, 1974 .

6. R.A. Kroeger, "Graduate Research Program in Aeronauti s or the Aerodynamics of Stall/ Incipient Spin," Second Semi-Annual Status Report, NASA Grant NSG-2018, The University of Michigan, 1975.

7. P.T. Wooler et al., "V/STOL Aircraft Aerodynamic Prediction Methods Investigation," USAF Tech. Rept. AFFDL-TR-72, Vol. 1, January, 1972.

8. E.F. Weener, "Stalled and Partially Stalled High Aspect Ratio, Straight Wings," Doctoral Dissertation, The University of Michigan, 1975.

9. Jaan Liiva, "Unsteady Aerodynamic and Stall Effects on Helicopter Rotor Blade Airfoil Sections," AIAA Paper 68-58, January, 1968.

10. R.M. Hicks and Vanderplaats, "Design of Low Speed Airfoils by Numerical Optimization," SAE Paper 750524, 1975. 